\title{
Paradigmenwechsel im Schweizer Gesundheitswesen
}

\author{
Das immer komplexer werdende Gesundheitswesen ist mit einer akteurzen- \\ trierten Sichtweise bald nicht mehr zu bewältigen. Patientenzentrierte und \\ ablaufoptimierte Lösungen rücken immer mehr in den Vordergrund [1].
}

Serge Reichlin

\footnotetext{
1 Walter A. Das Schweizer Gesundheitswesen ist ein System. Schweiz Ärztezeitung. 2008;89;16:704-6.
}

Korrespondenz:

Dr. med. Serge Reichlin

Facharzt für Innere Medizin FMH

Siemens Schweiz AG

Head Business Development

Sector Healthcare

Freilagerstrasse 40

CH-8047 Zürich

serge.reichlin@siemens.com
Der in den letzten Jahren immer stärker wirkende Kostendruck im Schweizer Gesundheitswesen wurde innerhalb der aktuellen, akteurzentrierten Struktur kompensiert. Organisationen wurden gestrafft, Softwarewerkzeuge evaluiert und implementiert, um die bestehenden Prozesse abzubilden. Die Akteure agieren dabei durch die Rahmenbedingungen getrieben individuell rational und erschweren damit eine Gesamtpotentialnutzung des Systems. Die fortwährende Optimierung der Struktur unter den aktuellen Anreizen blockiert dabei Reformen für eine nachhaltige Effizienzsteigerung durch eine übergreifende Ablaufoptimierung. Weil die Akteure die heutige Komplexität des Gesundheitswesens noch beherrschen, sind die Anreize, eine neue Ordnung zu definieren, sehr gering.

\section{Manuel sur les transformations du système de santé suisse}

Le système de santé suisse est en profonde mutation. Les moteurs principaux à la base de ces transformations sont la concurrence croissante entre acteurs, les nouveaux modèles de financement et de conventions entre ceux qui fournissent des prestations et ceux qui les financent, de même que l'évolution démographique et épidémiologique observée dans notre société. Les adaptations apportées aux conditions cadres légales augmentent la pression sur les acteurs. Les hôpitaux, tous les fournisseurs de prestations, les assureurs-maladie et l'industrie devront, dans les 2 à 5 ans, orienter leurs stratégies et les processus organisationnels sur ces facteurs de changement.

Ces thèses forment la trame du livre qui vient de paraître, uniquement en allemand, sous le titre «Gesundheitswesen Schweiz im Umbruch» et dans lequel 40 experts de la recherche, des sciences et de la pratique examinent attentivement, au fil de 50 articles spécialisés, les caractéristiques du système de santé
Die Folge ist ein verzerrter Wettbewerb zwischen Leistungserbringern und Versicherern, zwischen Versicherern und Kantonen sowie zwischen Kantonen und Leistungserbringern (Abb. 1). Das führt zu heterogenen System- und Finanzierungslandschaften, ineffizienten Abläufen zwischen den Akteuren sowie zu einem stetig steigenden Organisations- und Regulationsaufwand, um das System handlungsfähig und finanzierbar zu halten. Damit verursachen die aktuellen Anreize ein immer komplexer werdendes Schweizer Gesundheitswesen, mit mehrheitlich kostentreibenden Kräften. Die aktuellen Strategien stossen dabei an ihre Grenzen und der Patient wird im akteurzentrierten Gesundheitswesen im Spannungsfeld der individuell rational entscheidenden Akteure immer weiter an den Rand gedrängt.

suisse tout en exposant en détail les modifications qui se produiront au cours des prochaines années. On y relève les questions centrales suivantes: qu'est-ce qui rendra le système de santé suisse plus complexe à l'avenir? Quelles seront les répercussions de cette complexité accrue et quels processus deviendront-ils vitaux? Quelles mesures permettront-elles de développer la vitalité aux fins d'anticiper l'augmentation de la complexité?

Les éditeurs de cet ouvrage, à savoir Willy Oggier, Andreas Walter, Serge Reichlin et Michael Egli, sont quatre personnalités disposant d'une expérience professionnelle de longue date dans les domaines les plus divers de la santé publique. Quelques auteurs ont rédigé pour le BMS un article sur un thème central du manuel. Nous présentons leurs réflexions à notre lectorat dans les numéros 15 à 18-19/2008 sous forme de série brève.

Vous trouverez de plus amples informations sur le manuel «Gesundheitswesen Schweiz im Umbruch» sous www.ehealthcare.ch 


\section{Komplexität nimmt zu}

Einige Akteure haben die Veränderungskräfte bereits erkannt. Sie nutzen ihre Vitalität, investieren in die Zukunft und bereiten sich auf ein neues System mit neuen Anreizen vor. Weg vom akteurzentrierten, hin zu einem ablaufoptimierten System. Die Frage, die sich dabei stellt, ist, wie lange die zunehmende Komplexität mit den aktuellen Strategien und Strukturen noch zu bewältigen ist. (Abb. 2)
In einer umfassenden Analyse, die im Handbuch «Gesundheitswesen Schweiz im Umbruch» [2] die Komplexität des Schweizer Gesundheitswesens beschreibt, konnten zwei Faktoren mit einer kurzfristigen und schon heute spürbaren Komplexitätszunahme identifiziert werden: Zum einen sind es die Aktivitäten der Versicherer, die einen Wettbewerb fördern, der aber durch die aktuellen Anreize weiterhin verzerrend wirkt. Und zum anderen der politische Entscheid für

\section{Abbildung 1}

Ausgangslage - verzerrter Wettbewerb unter den Akteuren

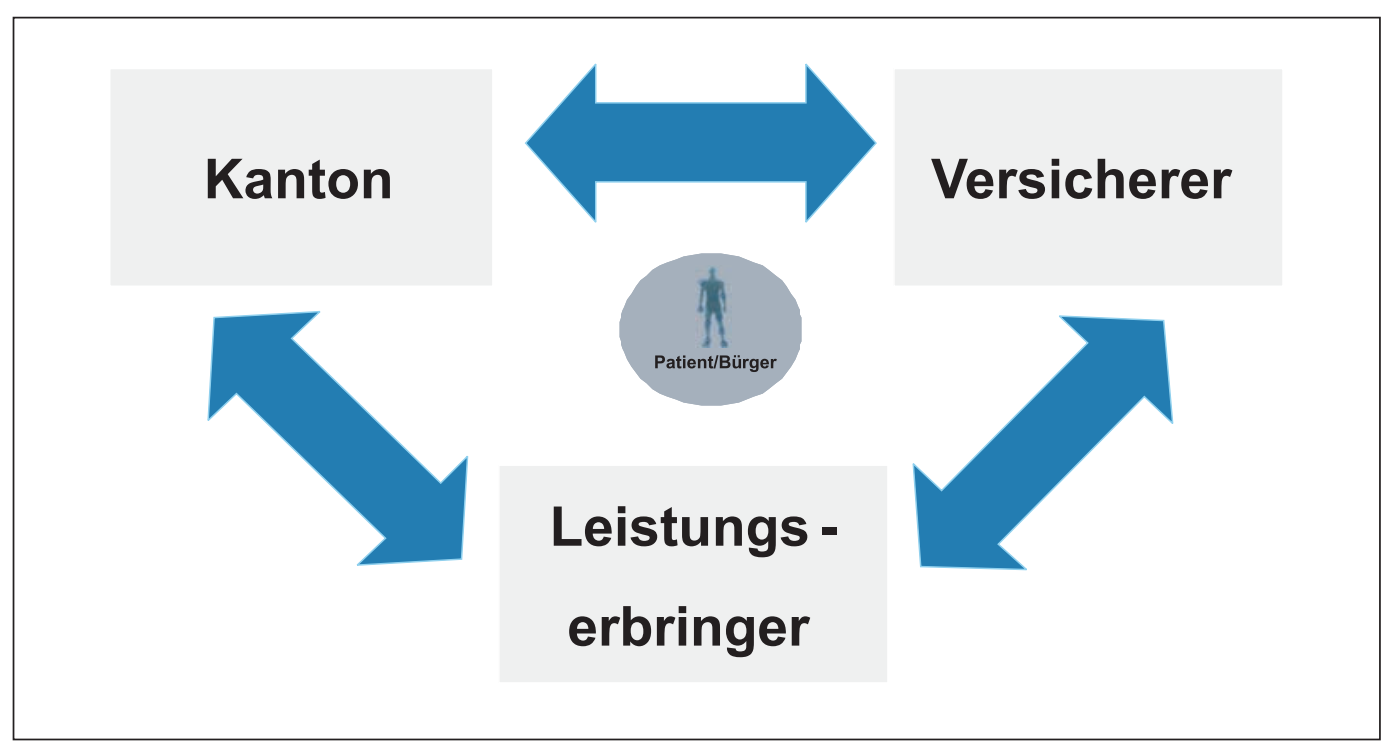

Abbildung 2

Der Druck der Komplexität wirkt auf die Vitalität der Akteure.

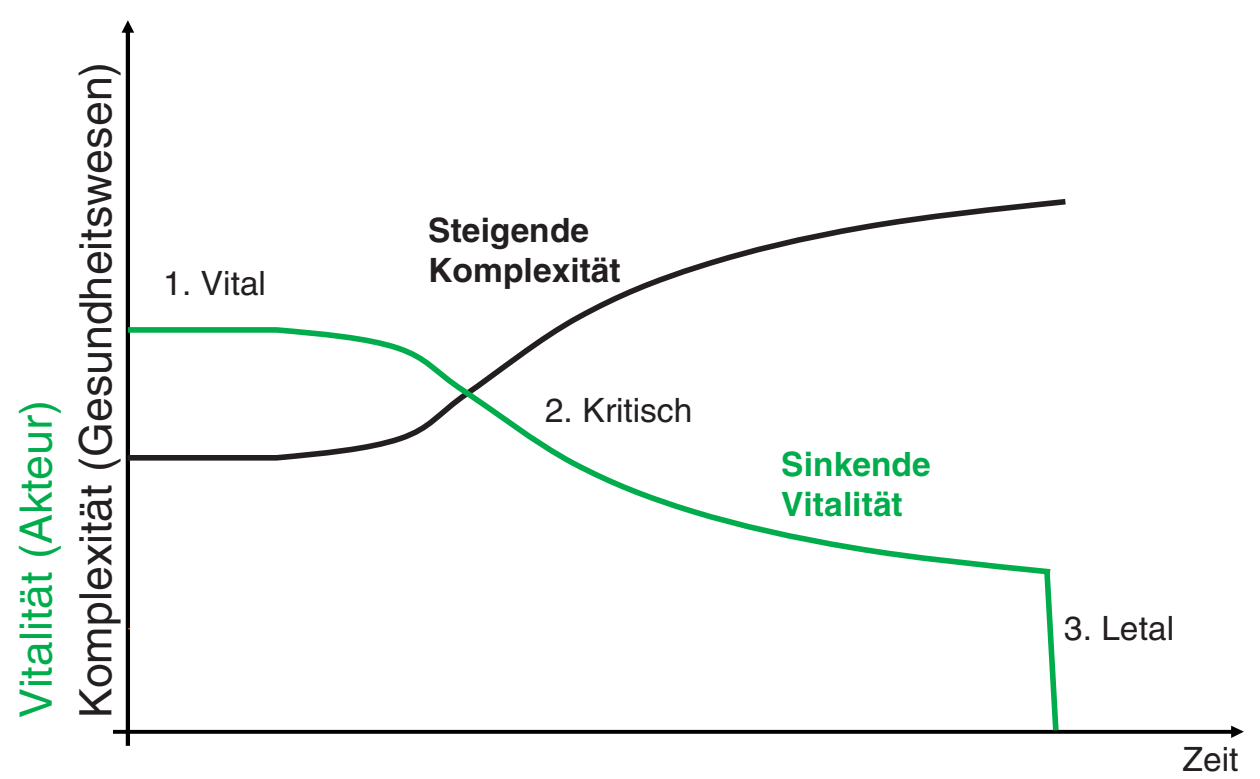


einen tiefgreifenden Wandel der Versorgungsstrukturen durch die Einführung der Leistungsfinanzierung.

Mittelfristig wird das System durch neue Vertrags- und Kooperationsmodelle zwischen Leistungserbringern und Leistungsträgern dynamisiert. Sie werden die Effizienz des Gesundheitswesens steigern, einen echten Wettbewerb unter den Akteuren verursachen und langfristig die Strukturen bedarfsgerecht ausrichten (Abb. 3). Neue Wettbewerbselemente wie ausgewiesene Qualität, höchste Spezialisierung bei gleichzeitiger Ganzheitlichkeit, Orientierung und vertrauensbildende Elemente, Krankheitsvermeidung beziehungsweise Sehrfrüherkennung bei gleichzeitiger Beherrschung der Mengenausweitung werden die Erfolgsfaktoren von morgen sein.

\section{Revisionspakete}

\section{haben hohe Abhängigkeiten}

Langfristig ist, vor allem durch die gestaffelte KVG-Revision, eine massive Erhöhung der Komplexität und eine Behinderung des Strukturumbaus zu erwarten. Um die politische Akzeptanz der notwendigen Revision zu erreichen, wurden die Reformen auf verschiedene Pakete verteilt. Die dadurch bedingte Entwicklung ist schwer abschätzbar, da die Revisionspakete hohe Abhängigkeiten untereinander aufweisen. Um die wesentlichen Systemanreize auf die Akteure für mehr Effizienz und Effektivität zu erzielen, müsste die Leistungsfinanzierung zusammen mit dem erweiterten Risikoausgleich unter den Kassen, der gleichgestellten Finanzierung und der Aufhebung des Kontrahierungszwangs gleichzeitig eingeführt werden.

Eine langfristige Zunahme der Komplexität konnte in der Analyse durch den Druck auf die Schweiz zur Anpassung an europäische Standards (z.B. die Pandemieszenarien) identifiziert werden. Die übergreifende Implementierung einer serviceorientierten Integrationsplattform oder das patientenfokussierte Ressourcenmanagement verringern die Komplexität nicht. Sie wirken jedoch, im Gegensatz zur gestaffelten KVG-Revision, als aktiv unterstützende Kraft auf dem Weg vom akteurzentrierten hin zum ablaufoptimierten Gesundheitswesen.

\section{Tiefgreifende Reformen sind nötig}

Schwer abzuschätzen sind die Auswirkungen des demographischen und epidemiologischen Wandels auf die Gesundheitsausgaben. Auch das Risiko, dass die Schweiz wegen ihrer Marktgrösse und -komplexität nicht immer zeitgerecht von Innovationen profitiert, kann zu einer Destabilisierung des Gesundheitssystems führen.

Die Gefahr ist gross, dass in Zukunft die Leistungsfähigkeit einzelner Leistungserbringer, aber auch der Versicherer durch die steigende Komplexität ernsthaft gefährdet sein könnte. Die heute bereits im Schweizer Gesundheitswesen wirkenden Kräfte werden den Wettbewerb und den transparenten Qualitätsausweis

Abbildung 3

Kooperative Modelle fördern echten Wettbewerb.

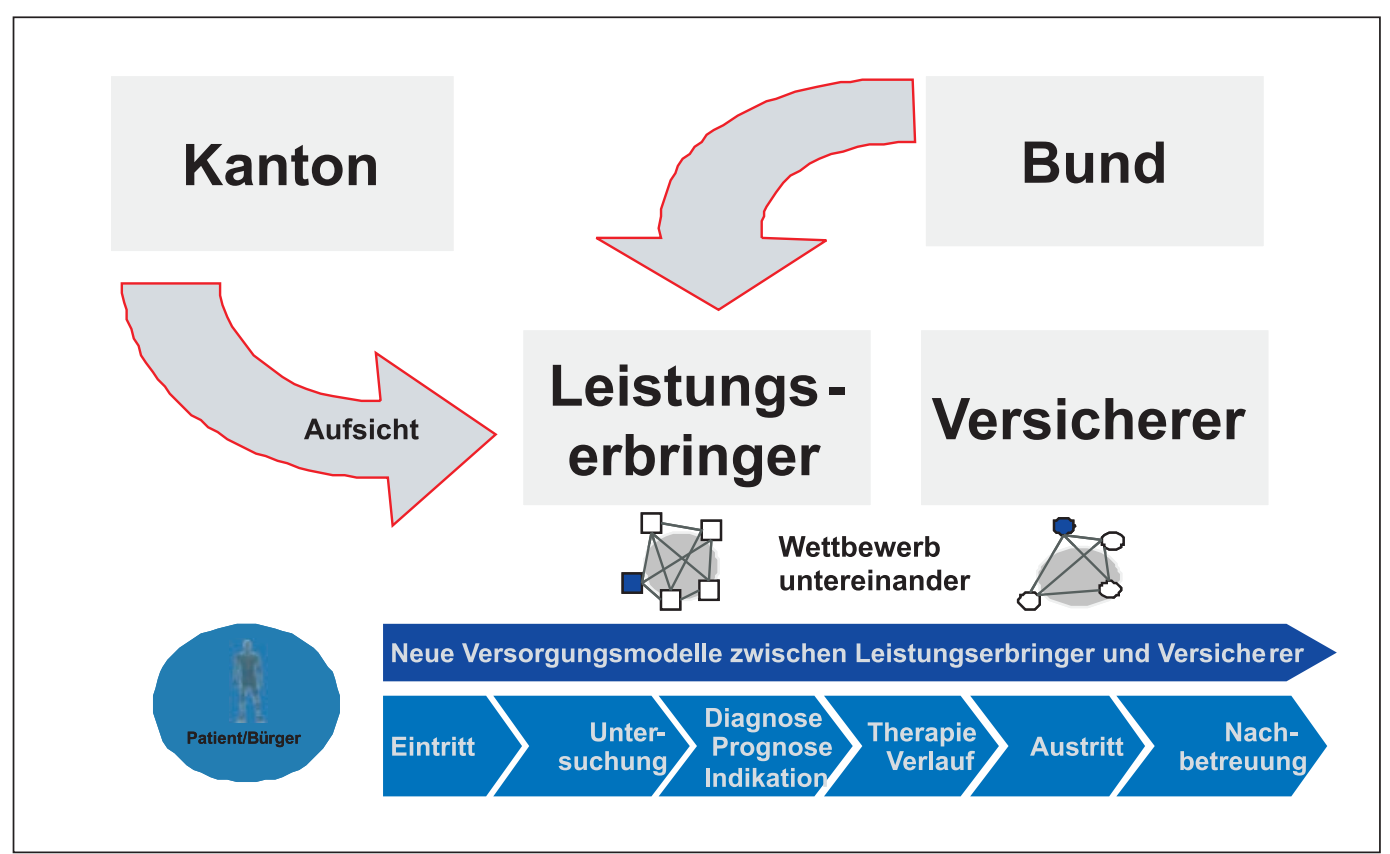


fördern und so einen Paradigmenwechsel mit patientenzentriertem und ablaufoptimiertem Fokus einleiten (Abb. 3).

\section{Auswirkungen auf die Leistungserbringer des Schweizer Gesundheitswesens}

Spitäler und Kliniken sind aktuell am stärksten den wirkenden Kräften im Schweizer Gesundheitswesen ausgesetzt. Dies zeigt sich vor allem darin, dass die meisten Wirkungsgrössen, wie im Handbuch «Gesundheitswesen Schweiz im Umbruch» [2] beschriebenen, diesen Akteuren zugeordnet werden können (Abb. 5).

Der eingeleitete Strukturumbau nach diagnosebezogener Versorgung, die zunehmende Wertigkeit des effektiven Diagnoseprozesses oder die Definition von wissensbasierten Behandlungspfaden sind zeitnah wirkende Kräfte (Abb. 4). Mittelfristig kommen die Wirkungen erster Akteure hinzu, die beginnen, ihre Strukturen ablaufoptimiert auszurichten, sowie die Wirkung budgetverantwortlicher CEOs und der steigende Wettbewerb mit der Folge einer notwendigen Differenzierungsstrategie. Mit der Wirkungsgrösse wettbewerbsfördernde Vertragsmodelle und dem Entscheid für die Einführung der Leistungsfinanzierung greifen nachhaltige Kräfte ein, die als Impulsgeber eine ganze Reihe von Wirkungsketten auslösen. Mit den Wirkungsgrössen Wettbewerbsvorteil durch Bilden und Managen von Versorgungsketten werden zwei zusätzliche Elemente angesteuert, die den Strukturwandel weiter antreiben.

Ähnlich wie die Spitäler steht auch die ambulante Grundversorgung unter grossem Druck der wirkenden Kräfte. Dieser Druck ist vor allem durch den Umbau der Spitalversorgung (Motor Strukturumbau) geprägt, der zusätzlich durch den inneren Strukturumbau des klassischen Hausarztansatzes angestossen wird. Die Versicherer fördern mit Wettbewerb und Vertragsmodellen diesen Trend, der sich vor allem in integrierten Versorgungsketten, in der Herausforderung der divergierenden Ziele zwischen Sehrfrüherkennung vs. Mengenausweitung und Spezialisierung vs. Ganzheitlichkeit ausdrückt. Die Regulatoren hemmen den Strukturumbau mit der gestaffelten KVG-Revision bei gleichzeitiger Komplexitätserhöhung.

Leistungserbringer werden mit Krankenversicherern, Regulatoren und der Industrie Ihre Strategie und die Leistungsprozesse in den nächsten 2-5 Jahren aufgrund dieser Veränderungskräfte konstruktiv diskutieren müssen. Der Paradigmenwechsel $\mathrm{zu}$ einem patientenzentrierten und ablaufoptimierten Schweizer Gesundheitssystem benötigt Kraft und Zeit. Wir werden unsere heutige Vitalität einsetzen müssen, wenn wir unsere Zukunft aktiv und im Sinne des Ganzen gestalten wollen.

\section{Abbildung 4}

Patientenzentrierter und ablaufoptimierter Strukturumbau.

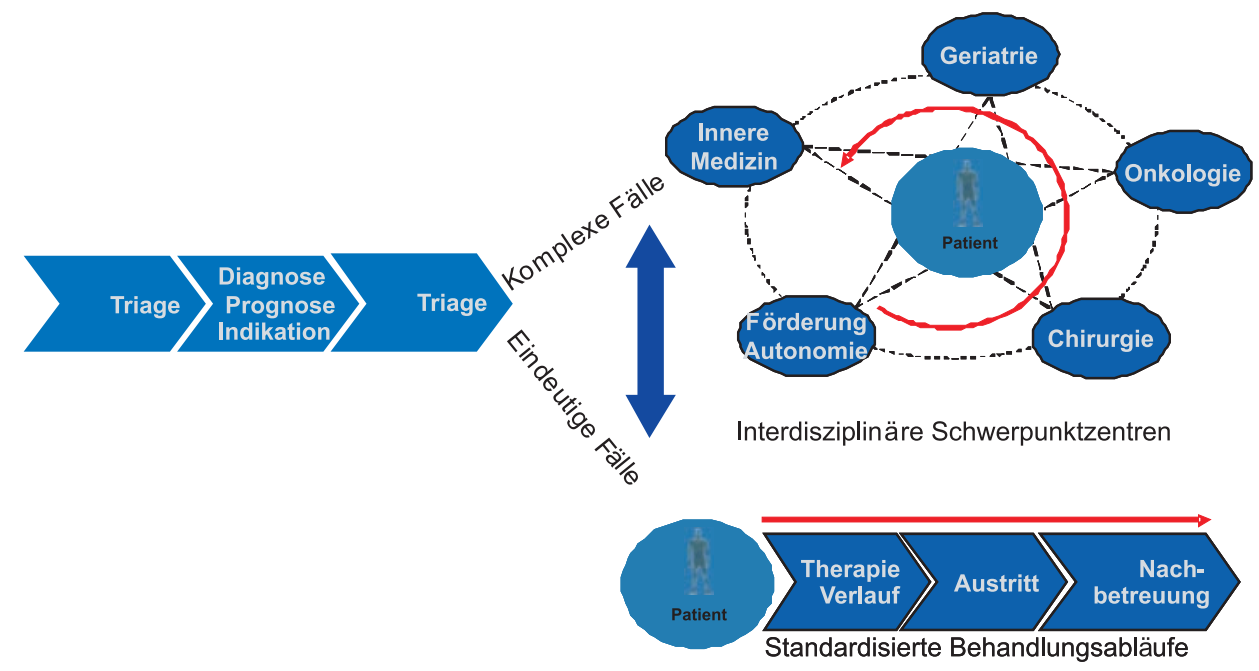




\section{Abbildung 5}

Leistungserbringer im Wirkungsgefüge des Schweizer Gesundheitswesens.

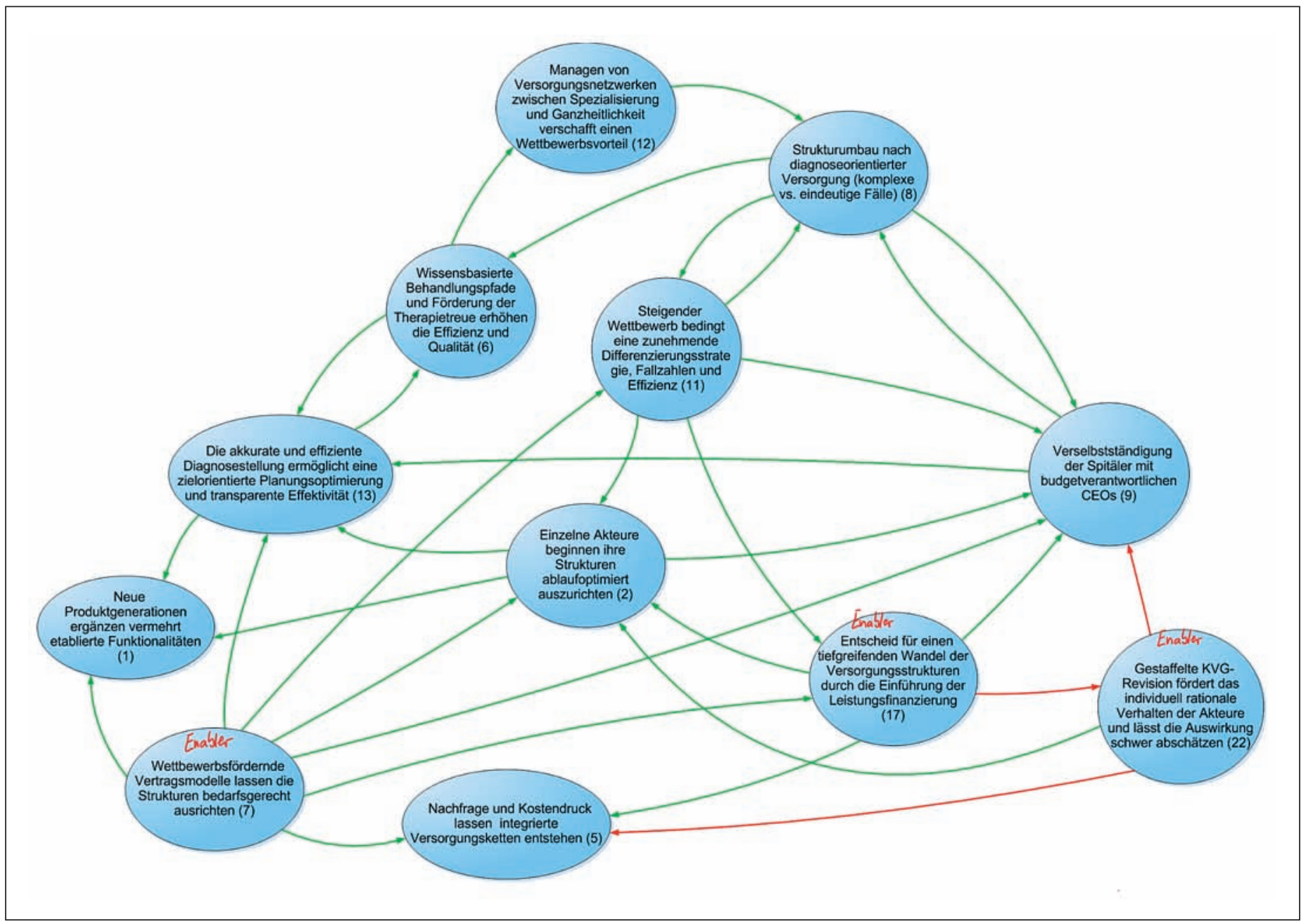

